\title{
Further Results on the Traveling Wave Solutions for an Integrable Equation
}

\author{
Chaohong Pan and Zhengrong Liu \\ Department of Mathematics, South China University of Technology, Guangzhou 510640, China \\ Correspondence should be addressed to Chaohong Pan; pan.ch@mail.scut.edu.cn
}

Received 19 December 2012; Accepted 8 February 2013

Academic Editor: Jong Hae Kim

Copyright ( 2013 C. Pan and Z. Liu. This is an open access article distributed under the Creative Commons Attribution License, which permits unrestricted use, distribution, and reproduction in any medium, provided the original work is properly cited.

The objective of this paper is to extend some results of pioneers for the nonlinear equation $m_{t}=(1 / 2)\left(1 / m^{k}\right)_{x x x}-(1 / 2)\left(1 / m^{k}\right)_{x}$ introduced by Qiao. The equivalent relationship of the traveling wave solutions between the integrable equation and the generalized $\mathrm{KdV}$ equation is revealed. Moreover, when $k=-(p / q)\left(p \neq q\right.$ and $\left.p, q \in \mathbb{Z}^{+}\right)$, we obtain some explicit traveling wave solutions by the bifurcation method of dynamical systems.

\section{Introduction}

Qiao [1] introduced the following equation:

$$
m_{t}=\frac{1}{2}\left(\frac{1}{m^{2}}\right)_{x x x}-\frac{1}{2}\left(\frac{1}{m^{2}}\right)_{x}
$$

which is the second positive member in a new completely integrable hierarchy. Equation (1) possesses a Lax representation and bi-Hamiltonian structure $[1,2]$. In $[1,2]$ the traveling wave solutions of (1) were studied. Sakovich [3] found the transformation

$$
x=v(y, t), \quad m(x, t)=\frac{-1}{v_{y}(y, t)},
$$

which relates (1) with the well-known modified KdV equation and obtained three types of smooth solitons of (1). Moreover, the equivalence of (1) and the modified CBS equation is proved in [4]. Yang and Chen [5] obtained two potentials and two pseudopotentials of (1). Equation (1) is derived from the two-dimensional Euler equation and proven to have Lax pair and bi-Hamiltonian structures [6].

To study the bifurcations of traveling wave solutions, $\mathrm{Li}$ and Qiao [7] considered the following nonlinear equation:

$$
m_{t}=\frac{1}{2}\left(\frac{1}{m^{k}}\right)_{x x x}-\frac{1}{2}\left(\frac{1}{m^{k}}\right)_{x}
$$

where $k \in \mathbb{R}, k \neq-1,0$. They used the phase analysis method of planar dynamical systems and the theory of the singular traveling wave systems [8-10] to find all possible bounded traveling wave solutions and their parametric representations for the cases of $|k|=1 / 2,2$, respectively. In fact, when $k=1 / 2$, (3) reads as a Harry Dym-type equation, which is actually the first member in the positive Camassa-Holm hierarchy [11-13]. The Harry Dym equation is an important integrable model in soliton theory [13]. It is also related to the classical string problem and has many applications in theoretical and experimental physics [14].

Sakovich [3] established the equivalent relationship between (1) and the modified KdV equation by the transformation for $k=2$. When $k \geq 2, k \in \mathbb{Z}$, one objective of this paper is to find some transformations which relate the traveling wave solutions of (3) with that of the generalized $\mathrm{KdV}$ equation [15]:

$$
u_{t}+a\left(u^{n}\right)_{x}+u_{x x x}=0,
$$

where $n$ is a positive integer and $a \neq 0$. For the results of traveling wave solutions on (4), we refer the readers to [1621]. The other objective is to extend the results of $\mathrm{Li}$ and Qiao [7]. We continue to consider the problems on explicit traveling wave solutions of (3) and their bifurcations, but we do not use the theory of the singular traveling wave systems. Instead, we apply the transformations which transform (3) into a traveling wave system without singular straight line 
$[21,22]$. Then, by using the bifurcation method of dynamical systems [21-28], we obtain some explicit traveling wave solutions of (3) for the case of $k=-(p / q)(p \neq q$ and $p, q \in$ $\left.\mathbb{Z}^{+}\right)$. Not only the existence of these solutions are proved, but also their concrete expressions are presented.

The rest of the paper is organized as follows. In Section 2, we reveal the equivalent relationship of the traveling wave solutions between (3) and (4). In Section 3, various planar systems and their bifurcation phase portraits of (3) are given. We state the explicit traveling wave solutions of (3) and present their theoretical derivation in Section 4. Some conclusions are given in Section 5.

\section{Equivalent Relationship of (3) and (4)}

In order to study the equivalent relationship of the traveling wave solutions between (3) and (4), we transform both (3) and (4) into traveling wave systems.

First of all, we substitute $m=U(\xi)$ with $\xi=x-c t(c \neq 0)$ into (3). Then, we get

$$
-c U^{\prime}=\frac{1}{2}\left(\frac{1}{U^{k}}\right)^{\prime \prime \prime}-\frac{1}{2}\left(\frac{1}{U^{k}}\right)^{\prime},
$$

where $c$ is a constant wave speed. Letting $k=-s$ and integrating (5) once, we have

$$
-c U=\frac{1}{2}\left(U^{s}\right)^{\prime \prime}-\frac{1}{2} U^{s}+g,
$$

where $g$ is an integral constant. Letting

$$
U=\varphi^{1 / s}
$$

then (6) can be rewritten as

$$
-c \varphi^{1 / s}=\frac{1}{2} \varphi^{\prime \prime}-\frac{1}{2} \varphi+g .
$$

Letting $n=1 / s$, we have

$$
\varphi^{\prime \prime}+2 c \varphi^{n}-\varphi+2 g=0 .
$$

Next, we also transform (4) into traveling wave system. Substituting $u(x, t)=\psi(\eta)$ with $\eta=c^{1 / 3} x-c t$ into (4), we have

$$
-c \psi^{\prime}+a c^{1 / 3}\left(\psi^{n}\right)^{\prime}+c \psi^{\prime \prime \prime}=0 .
$$

Integrating (10) once and letting $a=2 c^{5 / 3}$ leads to

$$
\psi^{\prime \prime}+2 c \psi^{n}-\psi+g_{1}=0
$$

where $g_{1}$ is an integral constant.

Finally, according to (9) and (11), we know that, from the traveling wave solution (3), we can drive the solutions of (4) for $k \in \mathbb{Z}$. When $k \in \mathbb{Z}$, one can notice that the traveling wave system of (4) is more general than (9) because of the arbitrary coefficient $a$. However, the traveling wave solutions of (3) cannot be derived from the solutions of (4) for $k \notin \mathbb{Z}$. Next, we study the traveling wave solutions of (3) and their bifurcations for $k \notin \mathbb{Z}$.

\section{Planar Systems and Their Bifurcation Phase Portraits}

In this section, we derive the traveling wave systems of (3) for the different cases of $k$ and draw their bifurcation phase portraits which are the basis for constructing nonlinear wave solutions.

When $k=-p / q$, that is, $n=q / p$, putting $y=\varphi^{\prime}$ and $g=0$, from (9), we obtain the following planar system

$$
\begin{gathered}
\frac{d \varphi}{d \xi}=y, \\
\frac{d y}{d \xi}=\varphi-2 c \varphi^{q / p} .
\end{gathered}
$$

Letting

$$
\varphi=\phi^{p}
$$

we have

$$
d \varphi=p \phi^{p-1} d \phi
$$

Then, system (12) can be written as

$$
\begin{gathered}
\frac{p \phi^{p-1} d \phi}{d \xi}=y, \\
\frac{d y}{d \xi}=\phi^{p}-2 c \phi^{q} .
\end{gathered}
$$

Under the transformation $d \tau=d \xi / p \phi^{p-1}$, we have

$$
\begin{gathered}
\frac{d \phi}{d \tau}=y \\
\frac{d y}{d \tau}=p \phi^{2 p-1}-2 c p \phi^{p+q-1} .
\end{gathered}
$$

System (16) has the first integral

$$
H(\phi, y)=h,
$$

where

$$
H(\phi, y)=y^{2}-\phi^{2 p}+\frac{4 c p}{p+q} \phi^{p+q}
$$

Next, we discuss the phase portraits of system. (16) for two different cases of $p-q$.

(a) $p-q$ is even and $c>0$.

Letting

$$
f(\phi)=p \phi^{2 p-1}-2 c p \phi^{p+q-1}=p \phi^{p-1}\left(\phi^{p}-2 c \phi^{q}\right),
$$

we have

$$
f^{\prime}(\phi)=p(2 p-1) \phi^{2 p-2}-2 c p(p+q-1) \phi^{p+q-2} .
$$

Solving $f(\phi)=0$, we get

$$
\phi_{1}=0, \quad \phi_{2 \pm}= \pm\left(\frac{1}{2 c}\right)^{1 /(p-q)} .
$$




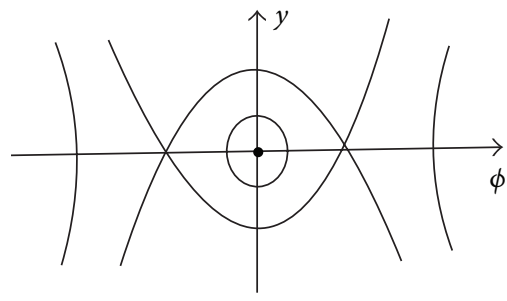

(a)



(d)

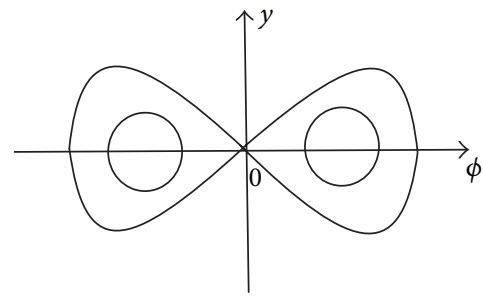

(b)

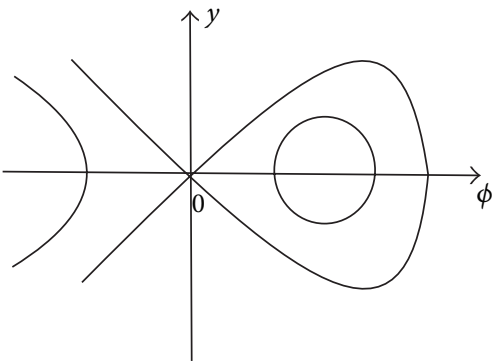

(e)

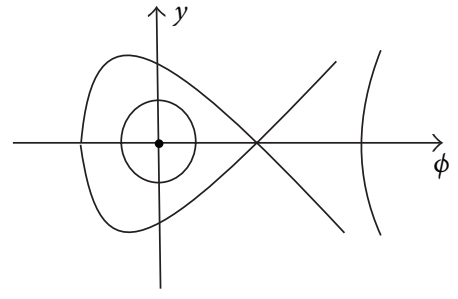

(c)

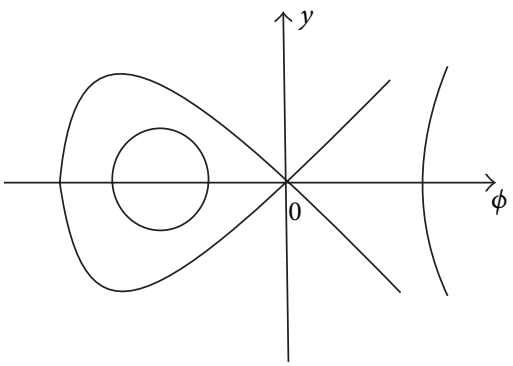

(f)

FIGURE 1: The phase portraits of Sy. (16). When $p-q$ is even and $c>0$, (a) and (b) are the phase portraits of system (16) for $p>q$ and $p<q$, respectively. When $p-q$ is odd, (c)-(f) are the phase portraits of system (16) for $p>q$ and $c>0, p>q$ and $c<0, p<q$ and $c>0, p<q$ and $c<0$, respectively.

At the singular point $(\phi, 0)$, it is easy to obtain that the linearized system of system (16) has the eigenvalues

$$
\lambda_{ \pm}(\phi, 0)= \pm \sqrt{f^{\prime}(\phi)} .
$$

From (12) and (18), we get the properties of the singular points $\left(\phi_{i}, 0\right)(i=1,2)$ as follow.

(i) If $f^{\prime}\left(\phi_{i}\right)>0$, then $\left(\phi_{i}, 0\right)$ is a saddle point of system (16).

(ii) If $f^{\prime}\left(\phi_{i}\right)<0$, then $\left(\phi_{i}, 0\right)$ is a center point of system (16).

(iii) If $f^{\prime}\left(\phi_{i}\right)=0$, then $\left(\phi_{i}, 0\right)$ is a degenerate singular point of system (16).

Therefore, we have the following results.

(1) When $p>q,\left(\phi_{1}, 0\right)$ is a center point, and $\left(\phi_{2+}, 0\right)$ and $\left(\phi_{2-}, 0\right)$ are saddle points. Due to $H\left(\phi_{2+}, 0\right)=$ $H\left(\phi_{2-}, 0\right)$, the orbits connecting with $\left(\phi_{2+}, 0\right)$ and $\left(\phi_{2-}, 0\right)$ are heteroclinic orbits.

(2) When $p<q,\left(\phi_{1}, 0\right)$ is a saddle point, and $\left(\phi_{2+}, 0\right)$ and $\left(\phi_{2-}, 0\right)$ are center points.

From the previous discussion, we get the phase portraits of system (13) as Figures 1(a) and 1(b).

(b) $p-q$ is odd

In this case, $f(\phi)$ has two zero points $\phi_{3}$ and $\phi_{4}$, where

$$
\phi_{3}=0, \quad \phi_{4}=\left(\frac{1}{2 c}\right)^{1 /(p-q)} .
$$

Similar to the previous discussion, we obtain the following results.
(1) When $p>q$ and $c>0,\left(\phi_{3}, 0\right)$ is a center point and $\left(\phi_{4}, 0\right)$ is a saddle point.

(2) When $p>q$ and $c<0,\left(\phi_{3}, 0\right)$ is a saddle point and $\left(\phi_{4}, 0\right)$ is a center point.

(3) When $p<q$ and $c>0,\left(\phi_{3}, 0\right)$ is a saddle point and $\left(\phi_{4}, 0\right)$ is a center point.

(4) When $p<q$ and $c<0,\left(\phi_{3}, 0\right)$ is a saddle point and $\left(\phi_{4}, 0\right)$ is a center point.

Through the discussion mentioned above, we obtain the phase portraits of Sy. (16) as Figures 1(c)-1(f).

\section{New Exact Solutions and \\ Theoretical Derivation}

When $k=-p / q, p \neq q, p, q \in \mathbb{Z}^{+}$, we have the following results.

(1) When $p>q$ and $p-q$ is odd, (3) has the following exact solution:

$m_{1}(x, t)$

$=\left[\frac{e^{-(x-c t)-X}}{16(p+q)^{2}}\left(e^{x-c t}+16 c p^{2} e^{X}+16 c p q e^{X}\right)^{2}\right]^{q /(p-q)}$,

where $X=q(x-c t) / p$.

(2) When $p>q, p-q$ is even and $c>0$, (3) also has the solution of the same expression as $m_{1}(x, t)$. 
(3) When $p<q$ and $p-q$ is odd, (3) has the solitary wave solution

$$
m_{2}(x, t)=\left[\frac{p+q}{4 c p} \operatorname{sech}^{2} \frac{(q-p)(x-c t)}{2 p}\right]^{q /(q-p)},
$$

and the blow-up solution

$$
m_{3}(x, t)=-\left[\frac{p+q}{4 c p} \operatorname{csch}^{2} \frac{(q-p)(x-c t)}{2 p}\right]^{q /(q-p)} \text {. }
$$

(4) When $p<q, p-q$ is even and $c>0$, (3) also has the solitary wave solution of the same expression as $m_{2}(x, t)$.

Next, we give the demonstration for the previous results of (3) by two cases.

Case $1(p<q)$. When $p<q, p-q$ is odd and $c>0$, system $(16)$ has two singular points $(0,0)$ and $\left(\phi_{2}, 0\right)$, where $\phi_{2}=(1 / 2 c)^{1 /(p-q)}$. The singular point $\left(\phi_{2}, 0\right)$ is a center point, and $(0,0)$ is a saddle point.

When $p<q, p-q$ is even and $c>0$, system (16) has three singular points $\left(\phi_{4}, 0\right),(0,0)$ and $\left(-\phi_{4}, 0\right)$, where $\phi_{4}=(1 / 2 c)^{1 /(p-q)}$. The singular points $\left(\phi_{4}, 0\right)$ and $\left(-\phi_{4}, 0\right)$ are center points, and the singular point $(0,0)$ is a saddle point.

Assume that $\left(\phi_{0}, 0\right)$ is an initial point of system (13), then we have the following results.

(1) When $0<\phi_{0} \leq \phi_{1}=(4 c p /(p+q))^{1 /(p-q)}$, the boundary of the closed orbit denoted by $\Gamma_{1}$ is a homoclinic orbit which passes $\left(\phi_{1}, 0\right)$ and connects with $(0,0)$ (see Figure 2(a)).

(2) When $\phi_{0}<0$, there are two special orbits denoted by $\Gamma_{2}^{+}$and $\Gamma_{2}^{-}$which pass through $(0,0)$ (see Figure $2(\mathrm{a})$ ).

(3) When $0<\phi_{0} \leq \phi_{3}$, we use $\Gamma_{3}$ to sign the orbit which passes through $\left(\phi_{3}, 0\right)$ and connects with $(0,0)$, where $\phi_{3}=(4 c p /(p+q))^{1 /(p-q)}$ (see Figure 2(b)).

On the $\phi-y$ plane, the orbits $\Gamma_{1}, \Gamma_{2}^{ \pm}$, and $\Gamma_{3}$ have the same expression which can be written as

$$
y= \pm \sqrt{\phi^{2 p}-\frac{4 c p}{p+q} \phi^{p+q}}
$$

Substituting the previous expression into $p \phi^{p-1} d \phi / d \xi=$ $y$ and integrating it along different orbits, we have

$$
\begin{aligned}
& \int_{\phi_{1}}^{\phi} \frac{p d s}{s \sqrt{1-(4 c p /(p+q)) s^{q-p}}}=|\xi| \quad\left(\text { along } \Gamma_{1}\right) \\
& \int_{-\infty}^{\phi} \frac{p d s}{s \sqrt{1-(4 c p /(p+q)) s^{q-p}}}=|\xi| \quad\left(\text { along } \Gamma_{2}^{ \pm}\right) \\
& \int_{\phi_{3}}^{\phi} \frac{p d s}{s \sqrt{1-(4 c p /(p+q)) s^{q-p}}}=|\xi| \quad\left(\text { along } \Gamma_{3}\right) .
\end{aligned}
$$

In (28), completing the integration and solving the equation for $\phi$, it follows that

$$
\phi_{2}(\xi)=\left[\frac{p+q}{4 c p} \operatorname{sech}^{2} \frac{(q-p) \xi}{2 p}\right]^{1 /(q-p)}
$$

Similarly, via (29) we have

$$
\phi_{3}(\xi)=-\left[\frac{p+q}{4 c p} \operatorname{csch}^{2} \frac{(q-p) \xi}{2 p}\right]^{1 /(q-p)} .
$$

Via (30), we get the solution of the same expression as $\phi_{2}(\xi)$.

When $p<q$ and $c<0$, we can obtain the solutions of the same expressions as $\phi_{2}(\xi)$ and $\phi_{3}(\xi)$.

Note that the transformation $m=\phi^{q}(\xi)$, we get the smooth solitary wave solution $m_{2}(x, t)$ and the blow-up solution $m_{3}(x, t)$ of (3).

Case $2(p>q)$. When $k=-p / q, p>q, p-q$ is odd and $c>0$, there is an interesting phenomenon concerning the traveling wave solutions of (3). Generally speaking, homoclinic orbit is corresponding to solitary wave solution. However, after applying the transformation to (3), we do not get solitary wave solution from homoclinic orbit. Therefore, we have the reason to believe that the transformation influences the corresponding relations between bifurcation orbits and traveling wave solutions.

(1) When $p>q, p-q$ is odd and $c>0$, system (16) has two singular points $(0,0)$ and $\left(\phi_{5}, 0\right)$, where $\phi_{5}=(2 c)^{1 /(p-q)}$. The singular point $(0,0)$ is a center point and the singular point $\left(\phi_{5}, 0\right)$ is a saddle point. The boundary of the closed orbit denoted by $\Gamma_{4}$ is a homoclinic orbit which passes through $\left(\phi_{6}, 0\right)$ and connects with $(0,0)$, where $H(0,0)=H\left(\phi_{6}, 0\right)$ and $\phi_{6}=(4 c p /(p+q))^{1 /(p-q)}$ (see Figure 3(a)).

(2) When $p>q, p-q$ is even and $c>0$, Sy. (16) has three singular points $(0,0),\left(\phi_{8}, 0\right)$, and $\left(-\phi_{8}, 0\right)$, where $\phi_{8}=(2 c)^{1 /(p-q)}$. The singular points $\left(\phi_{8}, 0\right)$ and $\left(-\phi_{8}, 0\right)$ are saddle points. The singular point $(0,0)$ is a center point. Due to $H\left(\phi_{8}, 0\right)=H\left(-\phi_{8}, 0\right)$, the orbits connecting with $\left(\phi_{8}, 0\right)$ and $\left(-\phi_{8}, 0\right)$ are two heteroclinic orbits. Here we only consider two special orbits which pass through $\left(\phi_{7}, 0\right)$, where $\phi_{7}=$ $(4 c p /(p+q))^{1 /(p-q)}$. We denote the orbits by $\Gamma_{5}^{ \pm}$(see Figure 3(b)).

On $\phi-y$ plane, the orbit $\Gamma_{4}$ and the orbits $\Gamma_{5}^{ \pm}$have the same expression

$$
y= \pm \sqrt{\phi^{2 p}-\frac{4 c p}{p+q} \phi^{p+q}}
$$

We take $\left((4 c p /(p+q))^{1 /(p-q)}, 0\right)$, that is, $\left(\phi_{6}, 0\right)$ or $\left(\phi_{7}, 0\right)$ as an initial point for system (16). Substituting the previous 


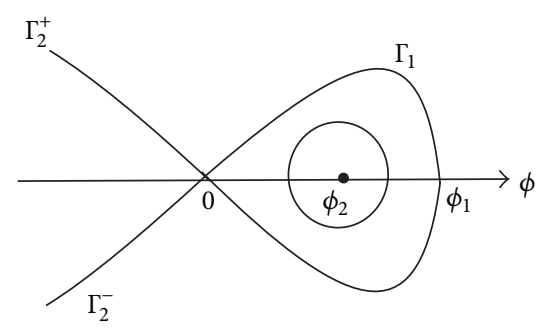

(a)

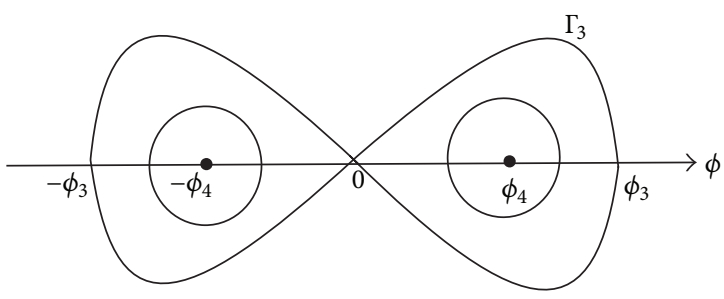

(b)

FIgURE 2: The phase portraits of Sy. (16). (a) For $p<q, p-q$ is odd and $c>0$. (b) For $p<q, p-q$ is even and $c>0$.

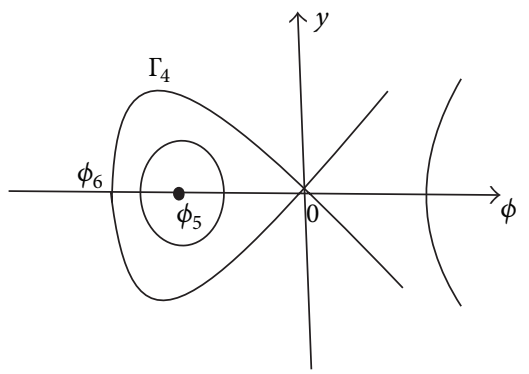

(a)

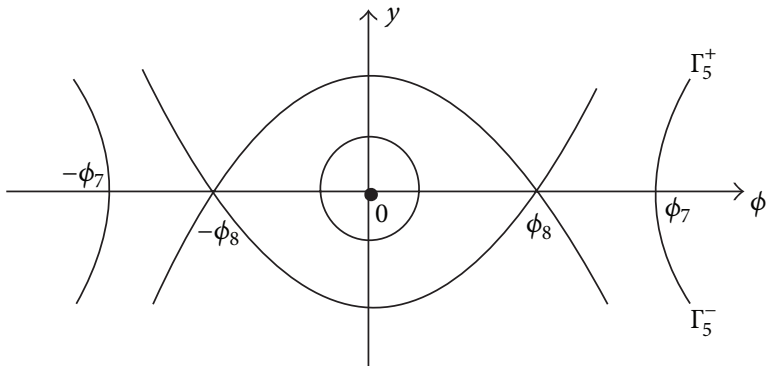

(b)

FIgURE 3: The phase portraits of Sy. (16). (a) For $p>q, p-q$ is odd and $c>0$. (b) For $p>q, p-q$ is even and $c>0$.

expression into $p \phi^{p-1} d \phi / d \xi=y$ and integrating it along $\Gamma_{4}^{ \pm}$ and $\Gamma_{5}^{ \pm}$, respectively, it follows that

$$
\begin{aligned}
& \int_{(4 c p /(p+q))^{1 /(p-q)}}^{\phi} \frac{p s^{p-1} d s}{\sqrt{s^{2 p}-4 c p /(p+q) s^{p+q}}} \\
& \quad=\int_{(4 c p /(p+q))^{1 /(p-q)}}^{\phi} \frac{p s^{(p-q) / 2} d s}{s \sqrt{s^{p-q}-4 c p /(p+q)}}=|\xi| .
\end{aligned}
$$

In (34), completing the integration and solving the equation for $\phi$, it follows that

$$
\phi_{1}(\xi)=\left[\frac{e^{-\xi-\eta}}{16(p+q)^{2}}\left(e^{\xi}+16 c p^{2} e^{\eta}+16 c p q e^{\eta}\right)^{2}\right]^{1 /(p-q)},
$$

where $\eta=q \xi / p$.

From the transformation $m=\phi^{q}(\xi)$, we obtain the traveling wave solution $m_{1}(x, t)$ of (3).

The theoretical derivation of the other cases can be finished similarly. We omit it for convenience. Hereto, we have completed the demonstrations to the previous results of (3).

\section{Conclusions}

In this paper, we find some transformations which relate (3) with (4). Applying these transformations, we reveal the relationship of traveling wave solutions between (3) and (4). By using the bifurcation method of dynamical systems, we consider the further results on the explicit traveling wave solutions of (3) for the special case of $k=-p / q$, where $p \neq q$, $p, q \in \mathbb{Z}^{+}$. The correctness of these solutions is tested as well by using the software Mathematica.

Note that in this paper, there are two problems waiting to solve. The first one is that we only discuss the equivalent relationship of the traveling wave solutions between (3) and (4). We do not know whether the relationship of the other solutions of (3) and (4) is equivalent. The second one is that we have investigated the traveling wave solutions of (3) for the special cases of $k=-p / q$. But the traveling wave solutions of (3) for the other cases of $k$ await further study.

\section{Conflicts of Interests}

The authors have declared that there is no conflict of interests.

\section{Acknowledgment}

This research is supported by the National Natural Science Foundation of China (no. 11171115), Natural Science Foundation of Guangdong Province (no. S2012040007959), and the Fundamental Research Funds for the Central Universities (no. 2012ZM0057).

\section{References}

[1] Z. J. Qiao, "New integrable hierarchy, its parametric solutions, cuspons, one-peak solitons, and M/W-shape peak solitons," Journal of Mathematical Physics, vol. 48, no. 8, pp. 082701082720, 2007. 
[2] Z. J. Qiao and L. P. Liu, "A new integrable equation with no smooth solitons," Chaos, Solitons and Fractals, vol. 41, no. 2, pp. 587-593, 2009.

[3] S. Sakovich, "Smooth soliton solutions of a new integrable equation by Qiao," Journal of Mathematical Physics, vol. 52, no. 2, p. 023509, 2011.

[4] P. G. Estévez, "Generalized Qiao hierarchy in 2+1 dimensions: reciprocal transformations, spectral problem and nonisospectrality," Physics Letters A, vol. 375, no. 3, pp. 537-540, 2011.

[5] Y.-Q. Yang and Y. Chen, "Prolongation structure of the equation studied by Qiao," Communications in Theoretical Physics, vol. 56, no. 3, pp. 463-466, 2011.

[6] Z. Qiao, "A new integrable equation with cuspons and W/Mshape-peaks solitons," Journal of Mathematical Physics, vol. 47, no. 11, pp. 112701-112709, 2006.

[7] J. Li and Z. Qiao, "Bifurcations of traveling wave solutions for an integrable equation," Journal of Mathematical Physics, vol. 51, no. 4, pp. 042703-042723, 2010.

[8] J. B. Li and H. H. Dai, On the Study of Singular Nonlinear Traveling Wave Equations: Dynamical System Approach, Science Press, Beijing, China, 2007.

[9] J. B. Li and G. Chen, "On a class of singular nonlinear traveling wave equations," International Journal of Bifurcation and Chaos in Applied Sciences and Engineering, vol. 17, no. 11, pp. 40494065, 2007.

[10] J. Li, J. Wu, and H. Zhu, "Traveling waves for an integrable higher order KdV type wave equations," International Journal of Bifurcation and Chaos in Applied Sciences and Engineering, vol. 16, no. 8, pp. 2235-2260, 2006.

[11] M. S. Alber, R. Camassa, Y. N. Fedorov, D. D. Holm, and J. E. Marsden, "The complex geometry of weak piecewise smooth solutions of integrable nonlinear PDE's of shallow water and Dym type," Communications in Mathematical Physics, vol. 221, no. 1, pp. 197-227, 2001.

[12] Z. J. Qiao, "The Camassa-Holm hierarchy, $N$-dimensional integrable systems, and algebro-geometric solution on a symplectic submanifold," Communications in Mathematical Physics, vol. 239, no. 1-2, pp. 309-341, 2003.

[13] M. Kruskal, "Nonlinear wave equations dynamical systems, theory and applications," Lecture Notes in Physics, vol. 38, pp. 310-354, 1975.

[14] M. J. Ablowitz and H. Segur, Solitons and the Inverse Scattering Transform, vol. 4, Society for Industrial and Applied Mathematics, Philadelphia, Pa, USA, 1981.

[15] R. M. Miura, "The Korteweg-de Vries equation: a survey of results," SIAM Review, vol. 18, no. 3, pp. 412-459, 1976.

[16] R. K. Dodd, J. C. Eilbeck, J. D. Gibbon, and H. C. Morris, Solitons and Nonlinear Wave Equations, Academic Press, London, UK, 1982.

[17] J. L. Bona, V. A. Dougalis, O. A. Karakashian, and W. R. McKinney, "Conservative, high-order numerical schemes for the generalized Korteweg-de Vries equation," Philosophical Transactions of the Royal Society of London A, vol. 351, no. 1695, pp. 107-164, 1995.

[18] B. Fornberg and G. B. Whitham, "A numerical and theoretical study of certain nonlinear wave phenomena," Philosophical Transactions of the Royal Society of London A, vol. 289, no. 1361, pp. 373-404, 1978.

[19] B. Dey, "Domain wall solutions of KdV-like equations with higher order nonlinearity," Journal of Physics A, vol. 19, no. 1 , pp. L9-L12, 1986.
[20] B. Dey, "KdV like equations with domain wall solutions and their Hamiltonians," in Solitons, Nonlinear Dynam., pp. 188194, Springer, Berlin, germany, 1988.

[21] Z. Liu and C. Yang, "The application of bifurcation method to a higher-order KdV equation," Journal of Mathematical Analysis and Applications, vol. 275, no. 1, pp. 1-12, 2002.

[22] M. Tang, R. Wang, and Z. Jing, "Solitary waves and their bifurcations of KdV like equation with higher order nonlinearity," Science in China A, vol. 45, no. 10, pp. 1255-1267, 2002.

[23] Z. Liu and T. Qian, "Peakons and their bifurcation in a generalized Camassa-Holm equation," International Journal of Bifurcation and Chaos in Applied Sciences and Engineering, vol. 11, no. 3, pp. 781-792, 2001.

[24] Z. Liu, A. M. Kayed, and C. Chen, "Periodic waves and their limits for the Camassa-Holm equation," International Journal of Bifurcation and Chaos in Applied Sciences and Engineering, vol. 16, no. 8, pp. 2261-2274, 2006.

[25] Z. R. Liu and Y. Long, "Compacton-like wave and kinklike wave of GCH equation," Nonlinear Analysis. Real World Applications, vol. 8, no. 1, pp. 136-155, 2007.

[26] Z. Liu and Z. Ouyang, "A note on solitary waves for modified forms of Camassa-Holm and Degasperis-Procesi equations," Physics Letters A, vol. 366, no. 4-5, pp. 377-381, 2007.

[27] M. Song and Z. Liu, "Periodic wave solutions and their limits for the generalized KP-BBM equation," Journal of Applied Mathematics, vol. 2012, Article ID 363879, 25 pages, 2012.

[28] Z. R. Liu, T. Jiang, P. Qin, and Q. Xu, “Trigonometric function periodic wave solutions and their limit forms for the KdVlike and the PC-like equations," Mathematical Problems in Engineering, vol. 2011, Article ID 810217, 23 pages, 2011. 


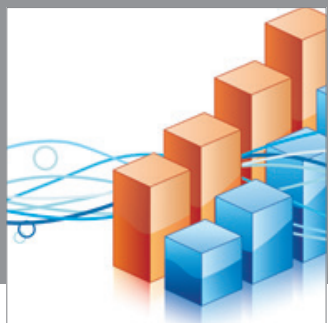

Advances in

Operations Research

mansans

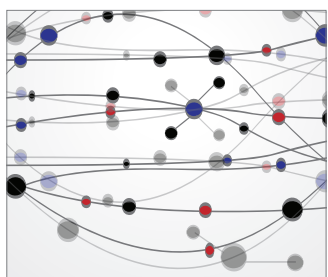

The Scientific World Journal
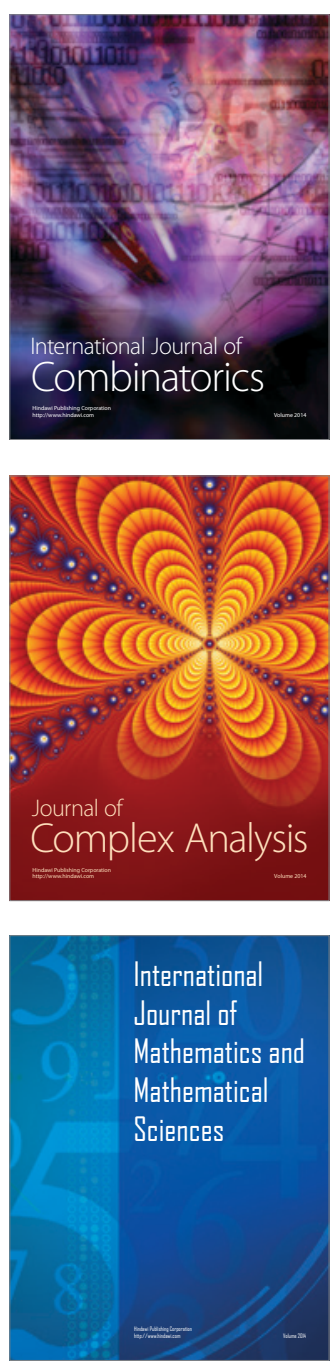
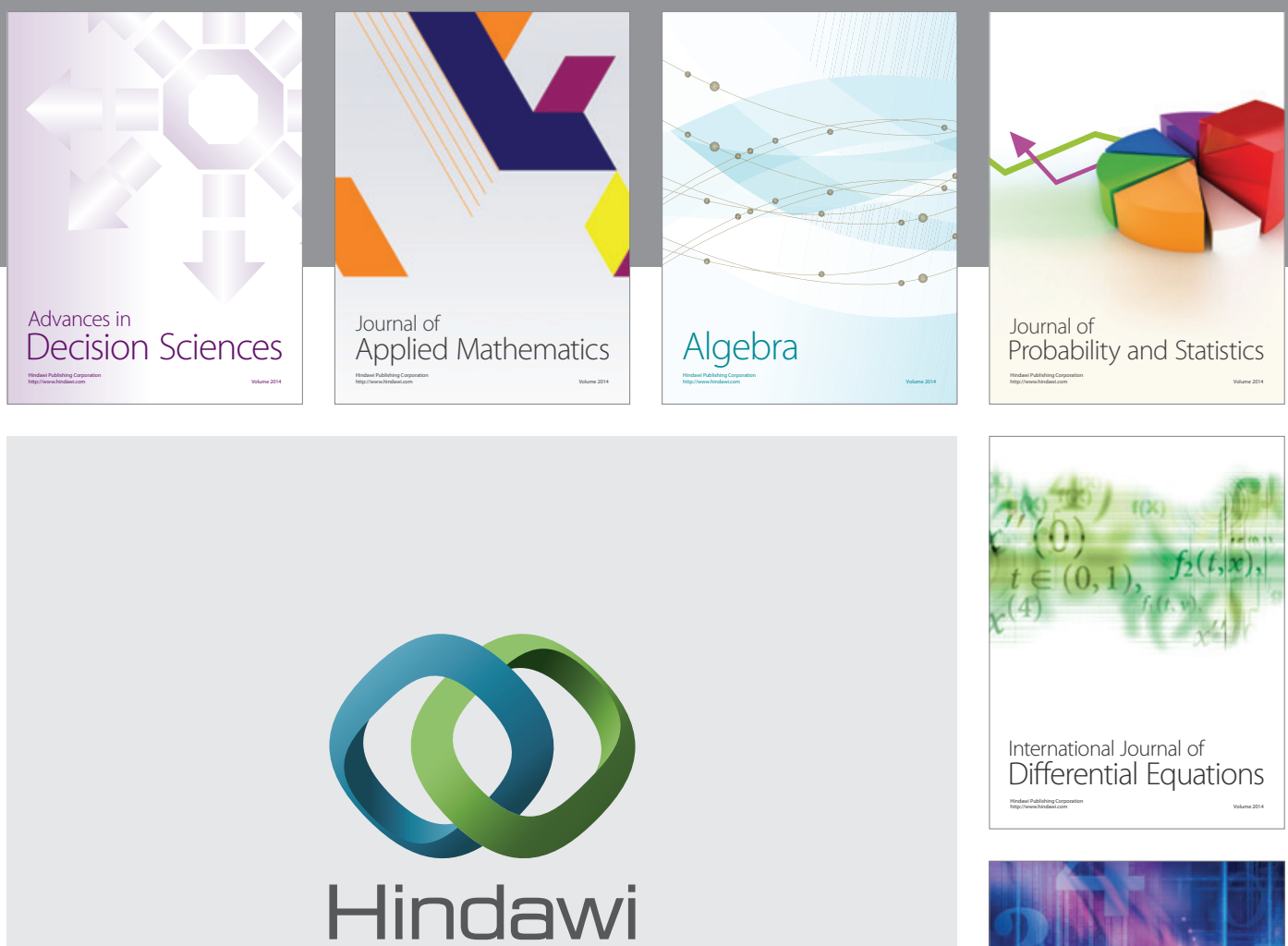

Submit your manuscripts at http://www.hindawi.com


Journal of

Function Spaces

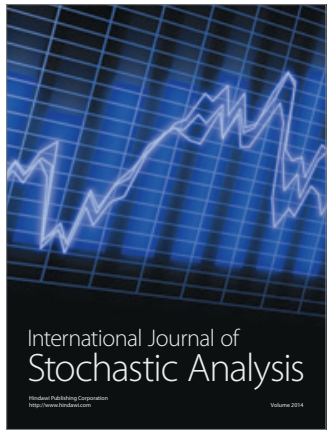

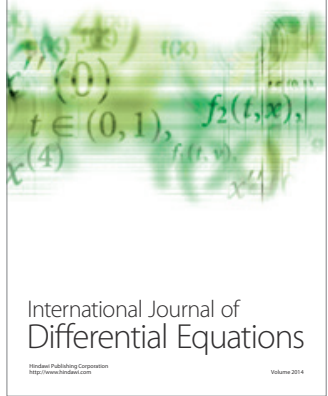
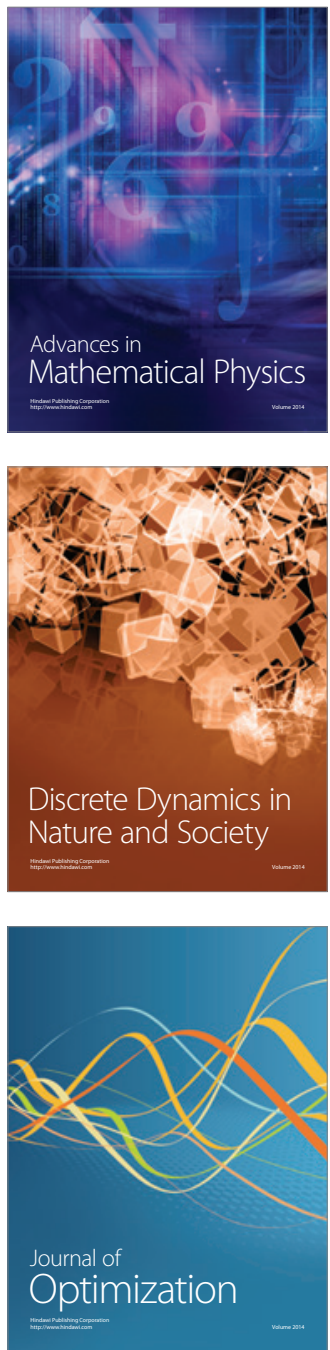